\title{
Interleukin-1 $\beta$-Induced Senescence Promotes Osteoblastic Transition of Vascular Smooth Muscle Cells
}

\author{
Linzi Han ${ }^{a, b}$ Yuying Zhanga, ${ }^{a}$ Mingming Zhang ${ }^{b}$ Liyu Guo ${ }^{a}$ \\ Jun Wang ${ }^{b}$ Fanjun Zeng ${ }^{a}$ Deping $\mathrm{Xu}^{c}$ Zongzhi Yin ${ }^{d}$ Yuanhong $\mathrm{Xu}^{\mathrm{c}}$ \\ Deguang Wang ${ }^{b}$ Haisheng Zhou a, e \\ aDepartment of Biochemistry and Molecular Biology, Anhui Medical University, Hefei, \\ China; ${ }^{b}$ Department of Nephrology, The Second Affiliated Hospital of Anhui Medical \\ University, Hefei, China; ${ }^{c}$ Clinical Laboratory, The First Affiliated Hospital of Anhui Medical \\ University, Hefei, China; d Department of Obstetrics and Gynaecology, The First Affiliated

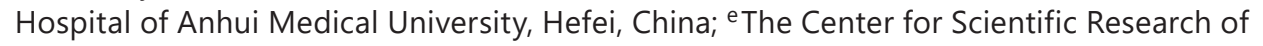 \\ Anhui Medical University, Hefei, China
}

\section{Keywords}

Interleukin-1 $1 \beta$ Senescence $\cdot$ Osteoblastic transition · Vascular calcification

\begin{abstract}
Introduction: Interleukin (IL)-1 $1 \beta$, as a key biomarker and mediator of vascular calcification in patients with end-stage renal disease (ESRD), may be involved in the process of premature senescence of vascular smooth muscle cells (VSMCs). This work sought to investigate whether IL-1 $\beta$-induced premature senescence contributes to the process of osteoblastic transition and vascular calcification in VSMCs. Methods: Eighty-eight patients with ESRD (aged 25-81 years), 11 healthy individuals, and 15 cases of lesion-free distal radial arteries from dialysis ESRD patients with angiostomy were collected in this study. Immunohistochemical analysis was performed to detect expression of IL-1 $\beta$, p21, and bone morphogenetic protein-2 (BMP2) in the distal radial arteries. Primary human VSMCs from healthy neonatal umbilical cords were incubated with test agents for 1-3 days. Intracellular levels of reactive oxygen species (ROS) and senescence-associated- $\beta$-galactosidase (SA- $\beta$-gal) staining were used to detect senescent cells. Alizarin red staining and the calcium content of the cell layer were used to detect mineral deposition in VSMCs. Results: Coincident with positive staining of IL-1 1 , p21, and BMP2 in the lesion-free distal radial arteries, $66.67 \%$ patients showed mineral deposition. Serum IL-1 $\beta$ was $0.24 \pm 0.57,1.20 \pm 2.95$, and $9.41 \pm 40.52 \mathrm{pg} / \mathrm{mL}$ in 11 healthy individuals, 20
\end{abstract}

L.H. and Y.Z. contributed equally to this work.

Dr. Deguang Wang

Department of Nephrology

The Second Affiliated Hospital of Anhui Medical University

No. 678 Furong Road, Hefei (China)

wangdeguang@ahmu.edu.cn
Dr. Haisheng Zhou

Department of Biochemistry and Molecular Biology

Anhui Medical University, No. 69 Meishan Road

Hefei (China)

haishengs@ahmu.edu.cn 


\section{Kidney \\ Blood Pressure \\ Research}

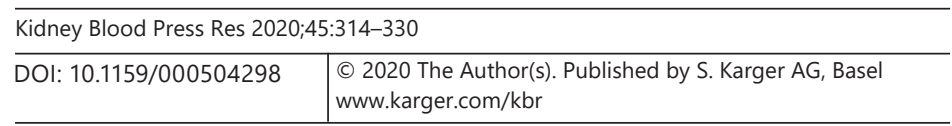

Han et al.: IL-1 $\beta$-Induced Senescence Promotes Osteoblastic Transition of VSMCs

patients without calcification, and 53 patients with calcification, respectively. Analysis of the cross-table chi-square test showed cardiovascular calcification is not correlated with levels of serum IL-1 $\beta$ in patients with ESRD $(p=0.533)$. In response to IL-1 $\beta$, VSMCs showed a senescence-like phenotype, such as flat and enlarged morphology, increased expression of p21, an increased activity of SA- $\beta$-gal, and increased levels of ROS. IL-1 $\beta$-induced senescence of VSMCs was required for the activation of IL-1 $3 / N F-k B / p 53 / p 21$ signaling pathway. IL-1 $\beta$ induced senescent VSMCs underwent calcification due to osteoblastic transition mainly depending upon the upregulation of BMP2. Resveratrol, an activator of sirtuin-1, postponed the IL-1 $\beta$-induced senescence through blocking the NF-KB/p53/p21 pathway and attenuated the osteoblastic transition and calcification in VSMCs. Conclusions: High levels of IL-1 1 in medial smooth muscles of arteries may play roles in inducing senescence-associated calcification. IL$1 \beta$-induced senescence depending on the activation of the NF-KB/p53/p21 signaling pathway and contributing to osteoblastic transition of VSMCs.

(C) 2020 The Author(s)

Published by S. Karger AG, Basel

\section{Introduction}

Chronic kidney disease (CKD) is highly correlated with cardiovascular mortality and vascular calcification. In anatomy, vascular calcification includes medial and intimal calcification. Intimal calcification, which is associated with atheromatous lipid-rich flow-limiting lesions, generally occurs in medium-sized conduit arteries with atherosclerotic plaques [1]. Medial calcification, associated with a diffuse granular pattern of mineral deposition, usually occurs in older population and patients with diabetic nephropathy $[2,3]$. Approximately $50 \%$ of patients with CKD show vascular calcification before hemodialysis [4]. Multiple recent studies have shown that disturbed phosphate/calcium metabolism, vascular smooth muscle cells (VSMCs) apoptosis, and osteogenic transition of VSMCs are associated with vascular calcification [4, 5]. In particular, inflammatory cytokines, including tumor necrosis factor- $\alpha$, interleukin (IL)- $1 \alpha$, IL-1 $1 \beta$, IL-4, and interferon- $\gamma$, play substantial roles in vascular calcification [6]. Among them, IL-1 $\beta$, a key biomarker and mediator of vascular calcification due to increased serum IL-1 $\beta$ levels in patients with chronic stable coronary heart diseases [7], has been considered to induce osteogenic transition of VSMCs in the presence of hyperphosphatemia [5, 8].

IL-1 $\beta$ is one of the most potent activators of the transcription factor NF- $\kappa$ B. In mammalian cells, classical NF- $\kappa B$ exhibits transactivation activities in the form of a heterodimer with p50 and $\mathrm{p} 65 /$ RelA proteins involved in inflammation and immune responses [9]. Activated NF- $\kappa \mathrm{B}$ is associated with the pathogenesis of CKD. Moreover, NF- $\mathrm{\kappa B}$ activation induced by a tumor necrosis factor-like weak inducer of apoptosis directly favors phosphorous-induced osteogenic transition and tissue nonspecific alkaline phosphatase (ALP) activity and promotes phosphorous-induced VSMC calcification [10].

Normal human cells undergo senescence in response to persistent DNA damage. There are no specific markers for senescent cells. Many cells express a $\mathrm{pH}$-labile enzyme called senescence-associated- $\beta$-galactosidase (SA- $\beta$-Gal) when they undergo senescence because of multiple stimuli. Cellular senescence is divided into 3 different types: replicative senescence, oncogene-induced senescence, and stress-induced senescence. Chronic stimulation of cells with cytokines causes enough stress to induce stress-induced senescence. Interestingly, there are some pieces of evidence that support correlation of stress-induced premature senescence with IL-1 $\beta$ stimulation $[11,12]$. In comparison with vehicle-treated controls, human osteoarthritic chondrocytes exposed to IL- $1 \beta$ exhibits an increased number of SA- $\beta$-Gal-positive cells [13]. Dai et al. [14] reported that the p53/p21 pathway mediates the features of chondrocyte senescence induced by IL-1 $\beta$ and hydrogen peroxide. 


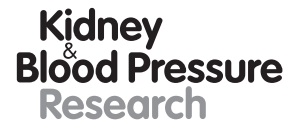

Kidney
Blood Pressure
Research

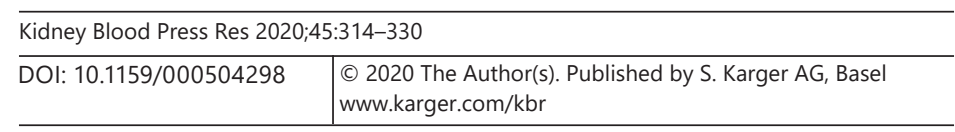

Han et al.: IL-1 $\beta$-Induced Senescence Promotes Osteoblastic Transition of VSMCS

As CKD is considered as an age-related disease, senescence-associated vascular calcification is becoming an interesting topic of study. Calcification of VSMCs is inhibited in the presence of macrophages and lipids [15], indicating the possible role of senescent VSMCs in the formation of vascular calcification. Compared with control young cells, VSMC calcification was reported to be dramatically enhanced in senescent cells. It is suggested that senescent VSMCs are apt to osteoblastic transition. Runt-related transcription factor-2 (RUNX2), an important transcription factor that regulates osteoblastic differentiation, is involved in the senescence-associated osteoblastic transition in VSMCs [16-18]. Bone morphogenetic protein-2 (BMP2), as a molecular marker of osteoblasts, is highly expressed in VSMCs in the process of osteoblastic transition.

However, little is known about senescence in VSMCs induced by IL-1 $\beta$. The involvement of IL-1 $\beta$ in the senescence-associated osteoblastic transition causing vascular calcification in VSMCs remains unclear. In addition, it remains to be determined whether pharmacological intervention can prevent senescence-associated osteoblastic transition that occurs in senescent VSMCs. In the present study, we examined whether plasma IL-1 $\beta$ coincided with vascular calcification caused by senescence-associated osteoblastic transdifferentiation of VSMCs in patients with end-stage renal disease (ESRD). We found that high levels of IL-1 $\beta$ in medial smooth muscles of arteries from patients with ESRD may play roles in inducing senescence-associated calcification. IL-1 $\beta$-induced senescence of VSMCs was required for the activation of the IL-1 $\beta / \mathrm{NF}-\kappa \mathrm{B} / \mathrm{p} 53 / \mathrm{p} 21$ pathway, and senescent VSMCs enhanced the susceptibility to calcification by increasing osteoblastic transition. Furthermore, we demonstrated that resveratrol effectively inhibited vascular calcification associated with osteoblastic transition in IL-1 $\beta$-induced senescent VSMCs.

\section{Materials and Methods}

\section{Patients}

Selected cases of ESRD patients were defined as albuminuria $\geq 300.0 \mathrm{mg} /$ day or estimated-glomerular filtration rate $<15 \mathrm{~mL} / \mathrm{min} / 1.73 \mathrm{~m}^{2}$. Plasma samples and the clinical data, including serum calcium, phosphate, and ALP levels and clinical images from patients with ESRD $(n=88)$, were collected at the Department of Nephrology in the Second Affiliated Hospital of AHMU between 2013 and 2015. The age of these patients ranged from 25 to 81 years. Among the patients with ESRD, 53 cases of patients were diagnosed using clinical images of vascular and valvular calcification obtained with computed tomography, plain $\mathrm{X}$-rays, or Doppler ultrasound imaging. Lesion-free distal radial arteries were separated from 15 cases of dialysis patients with angiostomy. The thoracic aorta was obtained from the cardiosurgical patients. According to the Chinese laws to treat human tissues and plasma left from surgery or clinical examination as discarded materials, informed consent for the experiments was not required.

Cytometrin Bead Analysis of Serum IL-1 $\beta$

The expression levels of IL- $1 \beta$ in the peripheral serum of patients with ESRD and healthy controls were detected using the Human IL-1 $\beta$ Detection Kit (Hangzhou Cellgene Biotech, CO., LTD, Hangzhou, China), according to the manufacturers' instructions.

\section{Primary Human VSMC Isolation and Cell Cultures}

Primary human VSMCs were obtained from a healthy neonatal umbilical cord and cultured in DMEM/F-12 medium (Hyclone, Logan, UT, USA) with 20\% (v/v) fetal bovine serum (FBS, Gibco BRL., Grand Island, NY, USA) and 1\% (v/v) penicillin-streptomycin (P/S) 


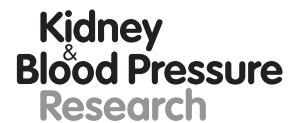

Kidney
Blood Pressure
Research

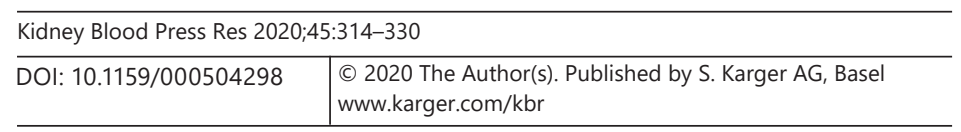

Han et al.: IL-1ß-Induced Senescence Promotes Osteoblastic Transition of VSMCs

at $37^{\circ} \mathrm{C}$ in a humidified atmosphere containing $5 \% \mathrm{CO}_{2}$. The medium was replaced with a fresh medium every 2-3 days. To induce senescence and calcification, 3-passage VSMCs were incubated in a calcification medium consisting of $3.8 \mathrm{~mm}$ sodium phosphate $(\mathrm{Pi}$, a mixture of $\mathrm{NaH}_{2} \mathrm{PO}_{4}$ and $\mathrm{Na}_{2} \mathrm{HPO}_{4}$, pH 7.4) and $10 \mathrm{ng} / \mathrm{mL}$ recombinant human IL-1 $\beta$ for $1-3$ days. Resveratrol $(0.1 \mathrm{mg} / \mathrm{mL})$ was supplemented to attenuate the senescence of VSMCs. To block IL-1 $\beta$ activities, $50 \mathrm{ng} / \mathrm{mL}$ anti-IL-1 $\beta$ neutralization antibodies (Sino Biological, Beijing, China) were supplemented in complete medium. Human endothelial cells (EAhy926) were cultured in DMEM with 10\% FBS and 1\% P/S. Primary human skin fibroblasts were cultured in DMEM/ F-12 medium supplemented with $20 \% \mathrm{FBS}$ and $1 \% \mathrm{P} / \mathrm{S}$.

\section{SA- $\beta$-Gal Staining}

To detect the cellular senescence in VSMCs, SA- $\beta$-Gal staining analysis was performed. Cells were fixed and stained with the SA- $\beta$-Gal staining kit (Beyotime Biotechnology, Shanghai, China) according to the manufacturer's protocol.

\section{Immunohistochemistry and Fluorescence Immunocytochemistry}

As described previously [19], immunohistochemistry (IHC) was performed to detect the target proteins using the streptavidin-peroxidase IHC kit (Origene, Beijing, China) according to the manufacturer's instructions. Primary antibodies against p21 (1:100; Cell Signaling Technology, CST, Danvers, MA, USA), IL-1 $\beta$ (1:300; Proteintech Group, Inc. Wuhan, China), and BMP2 (1:100; Novus Biologicals, Littleton, CO, USA) were used to stain the corresponding proteins, and IgG was used as a negative control. The sections were incubated with the diaminobenzidine Kit (Beyotime Biotechnology, Shanghai, China) to detect immune responses.

Fluorescence immunocytochemistry was used to detect expression of $\alpha$-smooth muscle actin ( $\alpha$-SMA), vimentin, and vascular endothelial growth factor receptor 2 (VEGFR2) in VSMCs. Briefly, cells were fixed with $4 \%$ paraformaldehyde, incubated overnight at $4{ }^{\circ} \mathrm{C}$ with the rabbit anti- $\alpha$-SMA antibody (1:200; Bioss, Beijing, China), the rabbit anti-vimentin antibody (1:100 dilution, CST, Danvers, MA, USA), or the rabbit anti-VEGFR2 antibody (1:400 dilution, Affinity Biosciences, Cincinnati, OH, USA), respectively. Then, the cells were washed and incubated with the anti-rabbit rhodamine-labeled secondary antibody (1:500; CST, Danvers, MA, USA). The cells were then counterstained with 4',6-diamino-2-phenyl indole (DAPI) and visualized under a fluorescence microscope.

\section{Calcification Staining}

Alizarin red staining was performed as previously described [20]. Briefly, VSMCs were washed twice with phosphate-buffered saline (PBS) and fixed with $4 \%$ formalin at room temperature for $20 \mathrm{~min}$. Cells were washed with PBS and stained with the Alizarin red stain kit (Solarbio., Beijing, China) to determine vascular calcification in VSMCs.

\section{Quantification of Calcium Deposition}

To evaluate calcium deposition, VSMCs were washed twice with PBS and decalcified with $0.6 \mathrm{~N} \mathrm{HCl}$ at $4{ }^{\circ} \mathrm{C}$ overnight. The calcium content of supernatants was determined with the calcium assay kit (Jiancheng Bioengineering Institute, Nanjing, China), according to the manufacturer's instructions. After calcium removal, the cells were washed 3 times with PBS, and proteins were extracted with the cell lysis buffer. The protein content was measured by the BCA protein assay kit (Jiancheng Bioengineering Institute, Nanjing, China). The calcium content of the cell layer was normalized to the protein content. 


\section{Kidney \\ Blood Pressure \\ Research}

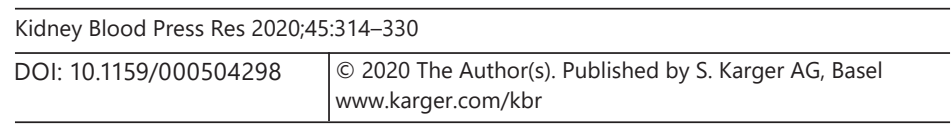

Han et al.: IL-1 $\beta$-Induced Senescence Promotes Osteoblastic Transition of VSMCs

Measurement of Intracellular Levels of Reactive Oxygen Species

The reactive oxygen species (ROS) assay kit (Solarbio., Beijing, China) was used to measure intracellular ROS according to manufacturer's instructions. Briefly, VSMCs were incubated with $2^{\prime}, 7^{\prime}$-dichlorodihydrofluorescein diacetate at $37^{\circ} \mathrm{C}$ for $20 \mathrm{~min}$, harvested, washed with PBS, and then detected immediately by flow cytometry. An increased minimum fluorescence intensity was used to quantify the generation of net intracellular ROS.

\section{Western Blotting Analysis}

A total $40 \mu \mathrm{g}$ of total proteins were obtained from cultured VSMCs, separated on $10 \%$ SDS-poly-acrylamide gel electrophoresis, and transferred onto a polyvinylidene fluoride membrane. After blocking with $5 \%$ non-fat milk for $1 \mathrm{~h}$ at room temperature, membrane with proteins was incubated overnight at $4{ }^{\circ} \mathrm{C}$ with primary antibodies against: sirtuin-1 (SIRT1; 1:1,000; CST, Danvers, MA, USA), p53 (1:1,000; CST, Danvers, MA, USA), acetylated-p53 (Ac-p53, Lys382; 1:1,000; CST, Danvers, MA, USA), p65 (1:1,000; CST, Danvers, MA, USA), phosphorlated-p65 (p-P65; 1:1,000; CST, Danvers, MA, USA), acetylated-p65 (Ac-p65, Lys310; 1:1,500; CST, Danvers, MA, USA), RUNX2 (1:1,500; Biorbyt, China), BMP2 (1:3,000; Novus Biologicals, Littleton, CO, USA), p21 (1:1,000; CST, Danvers, MA, USA), and GAPDH (1:5,000; Image, China). Blots were then incubated with peroxidase-conjugated secondary antibodies $(1: 5,000$; CST, Danvers, MA, USA) for $2 \mathrm{~h}$ at room temperature. Protein bands were detected using an enhanced chemiluminescence detection kit (Advansta, Menlo Park, CA, USA). Western blotting bands were captured by using the development instrument from Shanghai Qinxiang Scientific Instrument Co., Ltd.

\section{Statistics}

All statistical analyses were performed by SPSS (23.0, SPSS. Inc., Chicago, IL, USA). Normally distributed data were presented as mean \pm SD and analyzed using Student $t$ test or one-way ANOVA after confirming that the data passed the normality test. Non-normally distributed values were analyzed by the Kruskal-Wallis nonparametric test when the data failed to pass the normality test. $p<0.05$ was considered significant.

\section{Results}

Senescence was Consistent with Osteoblastic Transition and Calcification of VSMCs in the Patients with ESRD

The distal radial arteries from 15 patients with ESRD were collected and used to investigate the molecular coordination between the cellular senescence and vascular calcification associated with osteoblastic transition in VSMCs. Calcification staining (Alizarin red staining) was performed to determine calcium deposition in VSMCs. Immunohistochemical analysis of a senescence-related protein (p21) and an osteoblastic marker (BMP2) was performed with anti-p21 and anti-BMP2 antibodies, respectively. Muscular tunics in the thoracic aorta were negative for $\mathrm{p} 21$, BMP2, and calcium staining (Fig. 1A). Both $\mathrm{p} 21$ and BMP2 positively stained in and around the calcified area of the distal radial arteries from No. 2 and 4 patients with ESRD. Expression of IL-1 $\beta$, p21, and BMP2 and vascular calcification in the distal radial arteries from patients with ESRD are shown in Table 1 . Importantly, IL-1 $\beta$ was positively stained in the distal radial arteries from 12 ESRD patients. The clinical data showed that serum inorganic phosphate was $1.77 \pm 6.9 \mathrm{mM}$, serum calcium was $2.00 \pm 0.44 \mathrm{~mm}$, and ALP was $110.38 \pm 87.25 \mathrm{U} / \mathrm{L}$ in 15 patients with ESRD. Hypocalcemia $(<2.25 \mathrm{~mm})$ and hyperphosphatemia ( $>1.45 \mathrm{~mm}$ ) were observed in 9 patients. In addition, among these patients, only No. 1 patient had high level of serum ALP (392.00 U/L). Though clinical images showed cardio- 
Kidney

Blood Pressure

Research

\begin{tabular}{l|l}
\hline Kidney Blood Press Res 2020:45:314-330 \\
\hline DOI: 10.1159/000504298 & $\begin{array}{l}\text { @ 2020 The Author(s). Published by S. Karger AG, Basel } \\
\text { www.karger.com/kbr }\end{array}$ \\
\hline
\end{tabular}

Han et al.: IL-1 $\beta$-Induced Senescence Promotes Osteoblastic Transition of VSMCS

A

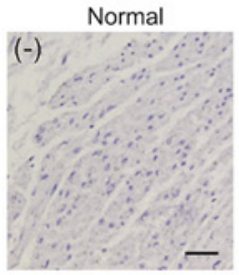

No.1 patient
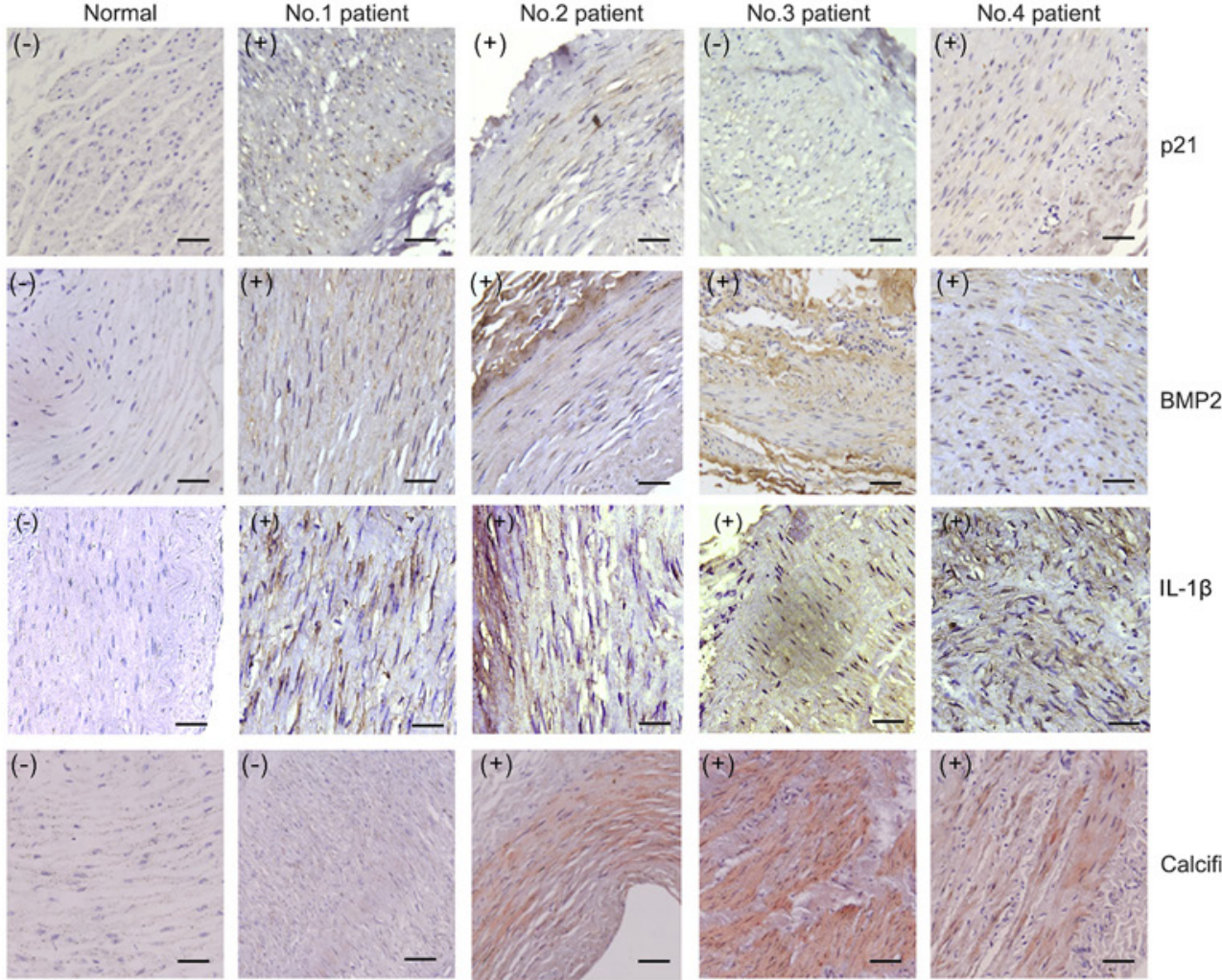

$\mathrm{IL}-1 \beta$

B
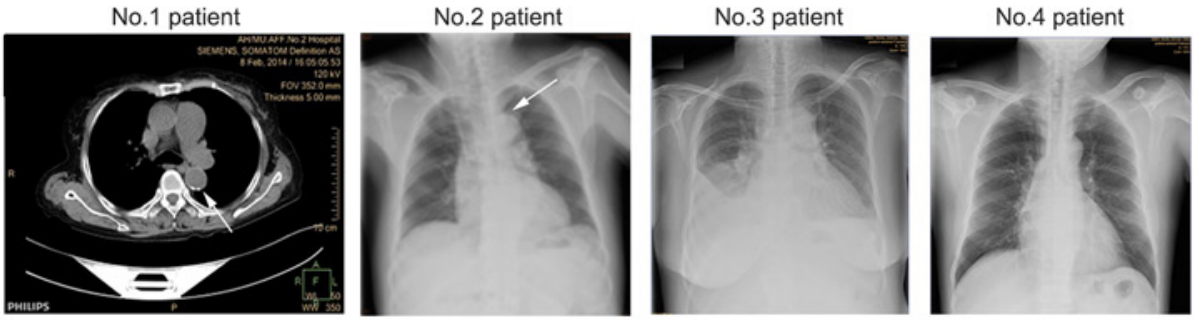

C

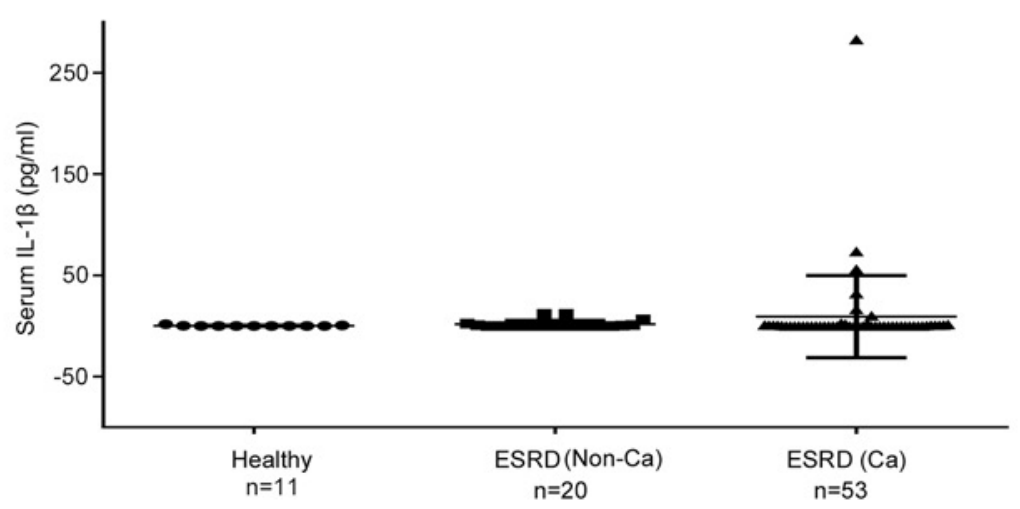


vascular calcification in 4 patients with ESRD (Fig. 1B, Table 1), 11 patients (73.3\%) showed mineral deposition in both BMP2- and p21-positive VSMCs of the distal radial arteries. Interestingly, coincident with positive staining of IL-1 $\beta, \mathrm{p} 21$, and BMP2 in the lesion-free distal radial arteries, 8 patients (66.67\%) showed mineral deposition.

To study the correlations between serum IL-1 $\beta$ and calcification, the clinical cardiovascular calcification images, including vascular calcification and valvular calcification, were collected from 73 cases of ESRD patients with serum determinations of IL-1 $\beta$. The plain $\mathrm{X}$-rays, CT imaging, and Doppler ultrasound imaging showed that cardiovascular calcification was found in 53 patients with ESRD (72.60\%, 53/73), whereas $27.4 \%$ of patients had no calcification (Fig. 1C, Table 2). Serum IL-1 $\beta$ was $0.24 \pm 0.57,1.20 \pm 2.95$, and $9.41 \pm 40.52 \mathrm{pg} / \mathrm{mL}$ in 11 healthy individuals, 20 patients without calcification, and 53 patients with calcification, respectively. Based on analysis of the cross-table chi-square test, cardiovascular calcification is not correlated with levels of serum IL-1 $\beta$ in patients with $\operatorname{ESRD~}(p=0.53)$.

Table 1. Clinical data of patients with ESRD and p21, BMP2, IL-1 $\beta$ expression, as well as mineral deposition in VSMCs

\begin{tabular}{|c|c|c|c|c|c|c|c|c|c|c|}
\hline $\begin{array}{l}\text { Number } \\
\text { of patients }\end{array}$ & Gender & $\begin{array}{l}\text { Age, } \\
\text { years }\end{array}$ & $\begin{array}{l}\text { Clinical } \\
\text { images of } \\
\text { calcification }\end{array}$ & $\begin{array}{l}\text { Serum } \\
\text { calcium, } \\
\mathrm{mm}\end{array}$ & $\begin{array}{l}\text { Serum } \\
\text { phosphate, } \\
\mathrm{mm}\end{array}$ & $\begin{array}{l}\text { ALP*, } \\
\text { U/L }\end{array}$ & $\begin{array}{l}\mathrm{IHC}^{\ddagger} \\
\text { for } \\
\text { p21 }\end{array}$ & $\begin{array}{l}\text { IHC for } \\
\text { BMP2 }\end{array}$ & $\begin{array}{l}\text { IHC for } \\
\text { IL-1 } \beta\end{array}$ & $\begin{array}{l}\text { Alizarin } \\
\text { red } \\
\text { staining }\end{array}$ \\
\hline 1 & Female & 76 & Yes & 1.91 & 1.82 & 392 & + & + & + & - \\
\hline 2 & Male & 76 & Yes & 1.65 & 1.25 & 90 & + & + & + & + \\
\hline 3 & Female & 50 & No & 2.15 & 0.57 & 98 & - & + & + & + \\
\hline 4 & Male & 39 & No & 1.59 & 2.11 & 97 & + & + & + & + \\
\hline 5 & Female & 64 & Yes & 1.91 & 0.84 & 98 & + & + & + & + \\
\hline 6 & Male & 33 & No & 1.96 & 2.22 & 83 & + & + & ND & + \\
\hline 7 & Male & 53 & Yes & 2.54 & 1.05 & 86 & + & + & + & + \\
\hline 8 & Male & 29 & No & 2.07 & 1.75 & 93 & + & + & + & + \\
\hline 9 & Female & 44 & No & 1.99 & 2.50 & ND & + & + & + & + \\
\hline 10 & Male & 30 & No & 2.03 & 1.60 & 58 & + & + & + & + \\
\hline 11 & Female & 50 & No & 1.06 & 3.09 & 125 & + & + & ND & + \\
\hline 12 & Female & 62 & No & 2.32 & 2.30 & 107 & + & + & + & - \\
\hline 13 & Female & 60 & No & 3.05 & 1.16 & 45 & + & + & + & + \\
\hline 14 & Male & 47 & No & 1.99 & 2.05 & 63 & + & - & + & - \\
\hline 15 & Male & 59 & No & 1.81 & 2.19 & ND & + & + & ND & + \\
\hline
\end{tabular}

Fig. 1. Cellular senescence is complicated by osteoblastic transition and vascular calcification in the distal radial arteries from the patients with ESRD. A Alizarin red staining for the detection of mineral deposition and immunohistochemistry analysis for expression of p21, BMP2, and IL-1 $\beta$ in VSMCs of the distal radial arteries from patients with ESRD (Scale bar $=200 \mu \mathrm{m}$ ). Normal indicates a lesion of thoracic aorta from a cardiosurgical patient. No. 1-4 denote lesion-free distal radial arteries from patients with ESRD. B Clinical images of No. 1-4 patients with ESRD. Arrows indicate the calcification sites of the thoracic aorta wall in No. 1 and 2 patients with ESRD. C Serum levels of IL-1 $\beta$ in the healthy and patients with ESRD. Non-Ca, patients without calcification; $\mathrm{Ca}$, patients with calcification; BMP2, bone morphogenetic protein-2; ESRD, end-stage renal disease; IL, interleukin. 


\section{Kidney \\ Blood Pressure \\ Research}

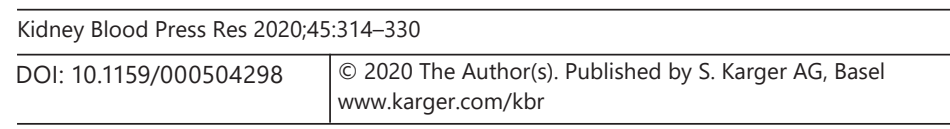

Han et al.: IL-1 $\beta$-Induced Senescence Promotes Osteoblastic Transition of VSMCs

\section{IL-1 $\beta$-Induced Premature Senescence of VSMCs}

IL-1 $\beta$ may be useful as a biomarker and predictor of vascular calcification risk in patients with ESRD [7] and may be involved in stress-induced premature senescence [11, 12]. In this study, primary human VSMCs were used as a cell model to investigate the molecular context of IL-1 $\beta$-induced senescence-associated osteoblastic transition and vascular calcification. Human VSMCs were isolated from the segments of the umbilical artery and cultured in DMEM/F-12 medium. To ensure the removal of spontaneous senescence, 3-day-cultured cells in the complete F12/DME medium were used as 3-day vehicle cells (control cells). Threepassage VSMCs were cultured for 1-3 days in complete DMEM/F-12 medium and displayed fusiform or ribbon-like shape under a microscope (Fig. 2A). VSMCs were further identified by fluorescence immunocytochemistry. a-SMA was successfully detected in both the 1 st and the 3rd passage VSMCs, whereas vimentin as well as VEGFR2 were not shown in VSMCs (Fig. 2B). Cells were cultured in DMEM/F-12 medium with sodium phosphate $(3.8 \mathrm{mM})$ in the absence or presence of IL-1 $\beta$ (10 ng/mL) to induce the senescence of VSMCs. As shown in Figure 2C, $D$, in comparison with hyperphosphate-treated controls, human VSMCs exhibited flat and enlarged morphology when exposed to IL-1 $\beta$ for 1-3 days. Furthermore, a dramatic increase in the SA- $\beta$-Gal activity was observed in VSMCs treated with IL-1 $\beta$ for 2-3 days. Resveratrol, a potent pharmacological agonist of SIRT1, was then used to attenuate the senescence of VSMCs. Compared to VSMCs in the calcification medium, both the cellular morphology and SA- $\beta$-Gal activity were not significantly altered in VSMCs treated with resveratrol or the antiIL-1 $\beta$ neutralization antibody. As a senescent marker, p21 was stably expressed in VSMCs cultured in medium with phosphate for 3 days (Fig. 2E). IL-1ßinduction dramatically enhanced p21 expression in VSMCs over 3 days. However, both resveratrol and the anti-IL-1 $\beta$ neutralization antibody markedly decreased p21 expression in VSMCs cultured in calcification

Table 2. Correlations between serum levels of IL-1 $\beta$ and calcification in the patients with ESRD

\begin{tabular}{lllll}
\hline $\begin{array}{l}\text { Serum IL-1 } \beta, \\
\mathrm{pg} / \mathrm{mL}\end{array}$ & \multicolumn{2}{l}{ Calcification (cases) } & Total, $\%$ & $p$ value \\
\cline { 2 - 4 }+ & + & - & \\
\hline 0 & 33 & 12 & $45(61.64)$ & 0.533 \\
$>0$ & 20 & 8 & $28(38.36)$ & \\
\hline Total, $\%$ & $53(72.60)$ & $20(27.40)$ & $73(100)$ & \\
\hline
\end{tabular}

ESRD, end-stage renal disease; IL, interleukin.

Fig. 2. IL-1 $\beta$ induces senescence of VSMCs. A Cellular morphology of VSMCs cultured in complete DMEM/F-12 medium for 0-3 days after 3 passages (Scale bar $=100 \mu \mathrm{m}$ ). B Fluorescence immunocytochemistry analysis of $\alpha$-SMA, vimentin, and VEGFR2 expression in the primary VSMCs, 3-passage VSMCs, human primary skin fibroblasts, as well as endothelial cells (EAhy926; Scale bar $=100 \mu \mathrm{m}$ ). C Cellular morphology of VSMCs treated with phosphate, IL-1 $\beta$, an anti-IL-1 $\beta$ neutralization antibody, or resveratrol over 3 days. (Scale bar = $100 \mu \mathrm{m})$. D SA- $\beta$-Gal activity detection in VSMCs by exposure to phosphate, IL-1 $\beta$, the anti-IL-1 $\beta$ neutralization antibody, or resveratrol over 3 days (Scale bar $=100 \mu \mathrm{m}$ ). E Immunoblotting analysis for p21 expression

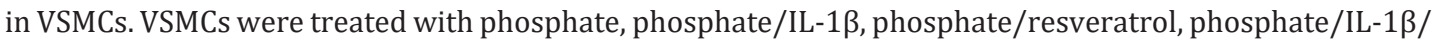
anti-IL-1 $\beta$ neutralization antibody, or phosphate/IL-1 $\beta /$ resveratrol over 3 days. F ROS levels of VSMCs with different treatments. The Y-axis denotes increased folds of the fluorescence intensity (ROS) comparing to the value of 3-day vehicle. Data were presented as the mean \pm SD of triplicate experiments. Pi, phosphate; Ab, anti-IL-1 $\beta$ neutralization antibody; Res, resveratrol; 3-day vehicle denotes 3-passage VSMCs cultured in complete DMEM/F-12 medium for 3 days; VSMC, vascular smooth muscle cell; $\alpha$-SMA, $\alpha$-smooth muscle actin; VEGFR2, vascular endothelial growth factor receptor 2; ROS, reactive oxygen species; IL, interleukin.

(For figure see next page.) 
medium with IL-1 $\beta$. Additionally, ROS levels in VSMCs treated with IL-1 $\beta$ were increased 1.4 folds than those in the 3-day-cultured cells (Fig. 2F). Whereas both resveratrol and the antiIL-1 $\beta$ neutralization antibody inhibited the increased ROS in VSMCs. It is suggested that IL-1 $\beta$ could generate the characteristics of premature senescent phenotypes, including altered cellular morphology, increased SA- $\beta$-Gal activity, increased p21 expression, as well as the increased ROS levels. Resveratrol has a role in delaying IL-1 $\beta$-induced senescence of VSMCs.

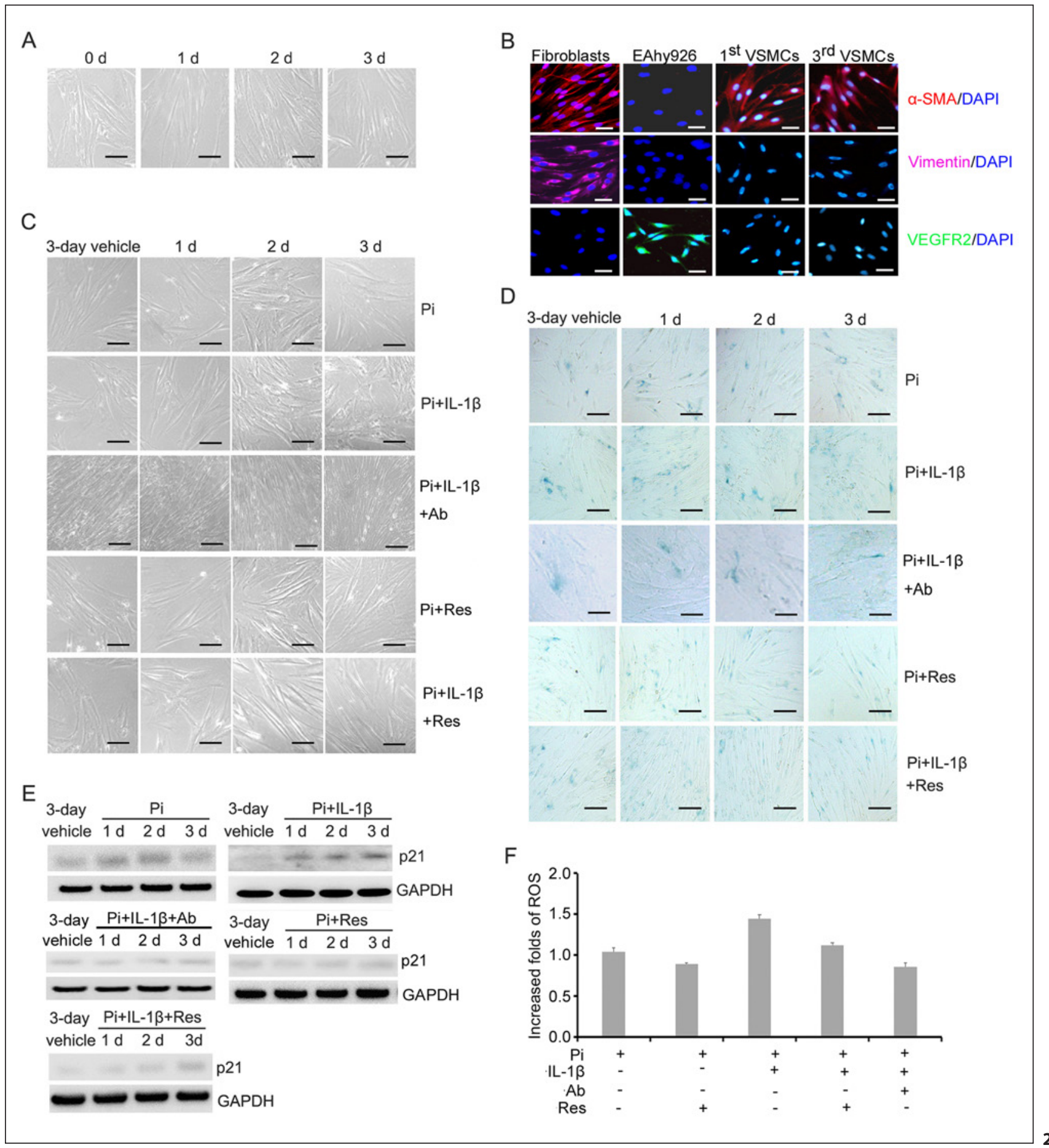




\section{Kidney \\ Blood Pressure \\ Research}

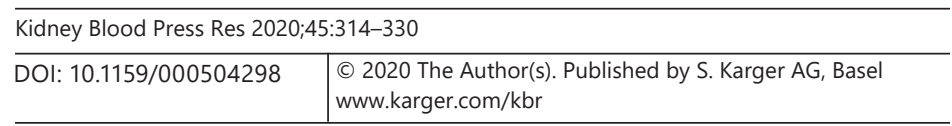

Han et al.: IL-1 $\beta$-Induced Senescence Promotes Osteoblastic Transition of VSMCs

IL-1 $\beta$-Induced Premature Senescence of VSMC through the NF- $\kappa B / p 53 / p 21$ Pathway

The transcription factor NF- $\mathrm{BB}$, activated by IL-1 $\beta$, has an important role in inflammation, immune responses, and pathogenesis of CKD [21]. It has been reported that crosstalk between $\mathrm{NF}-\kappa \mathrm{B}$ and SIRT1 is involved in many chronic metabolic and age-related diseases [22]. To further investigate the molecular context of IL-1 $\beta$-induced senescent VSMCs, the immunoblotting analysis was performed to determine the alterations of SIRT1 and activation of the NF- $\kappa B$ signaling pathway. As shown in Figure 3A, as an endogenous inhibitor of senescence, SIRT1 expression showed no significant changes in VSMCs cultured in calcification medium, even after exposure to IL-1 $\beta$. There were no obvious alterations of p-p65, Ac-p65, p53, as well as Ac-p53 in VSMCs cultured in calcification medium. The activated NF- $\mathrm{BB}$ (p-p65), Ac-p65, p53 (a downstream molecule of activation NF- $\kappa$ B), and Ac-p53 markedly increased in VSMCs in the presence of IL-1 $\beta$, compared with those of the 3-day vehicle cells. However, IL-1 $\beta$ induced the senescence of VSMCs through the activation of the NF- $\kappa B / p 53 / p 21$ pathway, which was dramatically inhibited by the anti-IL-1 $\beta$ neutralization antibody.

After treatment with resveratrol for 1 day, SIRT1 expression dramatically increased in VSMCs grown in the high phosphate medium (Fig. 3B). However, no significant change in Ac-p53 and p53 was observed in VSMCs on exposure to high phosphate medium with resveratrol over a 3-day period. SIRT1 and Ac-p65 gradually increased in VSMCs on exposure to high phosphate medium with resveratrol and IL-1 $\beta$ over 2 days. This suggests that resveratrol dramatically inhibited IL-1 $\beta$-induced senescence of VSMCs by activating SIRT1.

\section{IL-1ß-Induced Vascular Calcification in VSMCs}

To explore whether IL-1 $\beta$ is responsible for enhanced VSMC calcification in the senescent cells, Alizarin red staining was performed to detect calcium deposition in VSMCs. Similar to hyperphosphatemia-based arterial calcification in vivo, hyperphosphate-induced calcification spontaneously occurred in VSMCs. Interestingly, calcium deposition was dramatically enhanced in VSMCs by exposure to IL-1 $\beta$ over 3 days (Fig. 4A). However, either resveratrol or the antiIL-1 $\beta$ neutralization antibody effectively repressed hyperphosphate-induced and IL-1 $\beta$-induced calcification in VSMCs. Furthermore, calcium content in 3-day VSMCs was then determined with the calcium assay kit. Compared with 3-day vehicle cells, VSMCs showed a higher level of intracellular calcium induced by both phosphate and IL-1 $\beta$. Increased intracellular calcium was markedly inhibited by resveratrol or the anti-IL-1 $\beta$ neutralization antibody (Fig. 4B).

\section{IL-1 $\beta$-Induced Calcification was Required for Senescence-Associated Osteoblastic}

Transition in VSMCS

Further, to determine whether hyperphosphate-induced calcification is related to senescence-associated osteoblastic transition in VSMCs, the immunoblotting analysis was performed to detect the expression of RUNX2, BMP2, p21, and a-SMA in VSMCs cultured in calcification medium for 3 days. As shown in Figure 5A, no significant alterations of p21, a-SMA, and BMP2 were observed in VSMCs. But RUNX2 expression dramatically increased in VSMCs. a-SMA expression gradually increased in VSMCs on exposure to resveratrol over a 3-day period. However, coincident with a decreased p21 expression, RUNX2 and BMP2 expression dramatically decreased in VSMCs treated with resveratrol over 3 days.

Interestingly, the increased expression of BMP2 and RUNX2 was markedly observed in VSMCs treated with IL-1 $\beta$, which was coincident with increased p21 expression. But significant alterations of RUNX2 and BMP2 expression were not observed in VSMCs exposed to both IL-1 $\beta$ and resveratrol as compared to those of the vehicle cells (Fig. 5B). Taken together, IL-1 $\beta$-induced calcification was required for senescence-associated osteoblastic transition in VSMCs, and the senescence-associated osteoblastic transition was markedly inhibited by resveratrol. 
A

vehicle $\frac{P i}{1 d} \quad 2 d \quad 3 d$ vehicle $\frac{P i+I L-1 \beta}{1 d} 2 d \quad 3 d$ vehicle $\frac{P i+I L-1 \beta+A b}{1 d \quad 2 d \quad 3 d}$
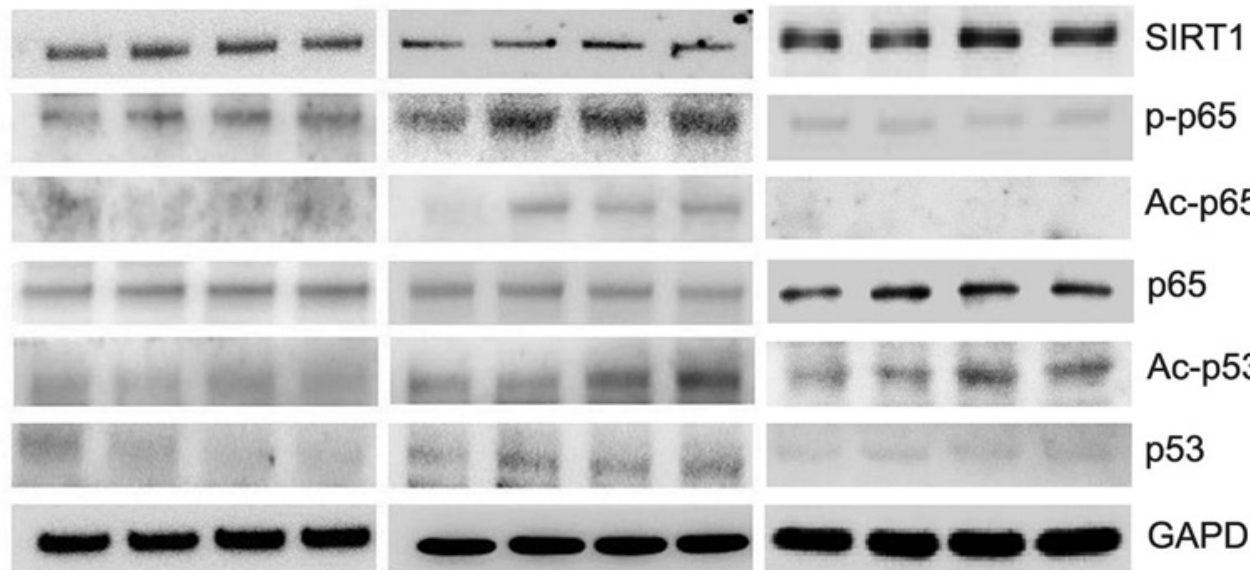

B

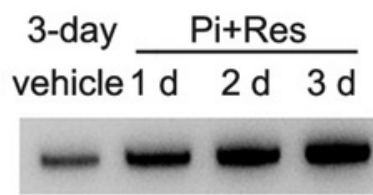

3-day $\mathrm{Pi}+\mathrm{IL}-1 \beta+\mathrm{Res}$
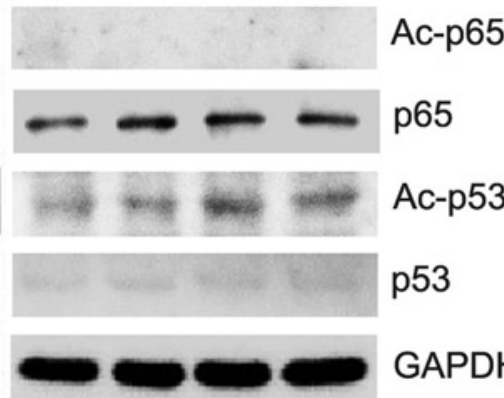

p53

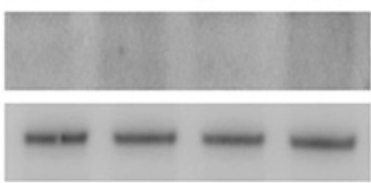
vehicle $1 \mathrm{~d} \quad 2 \mathrm{~d} \quad 3 \mathrm{~d}$
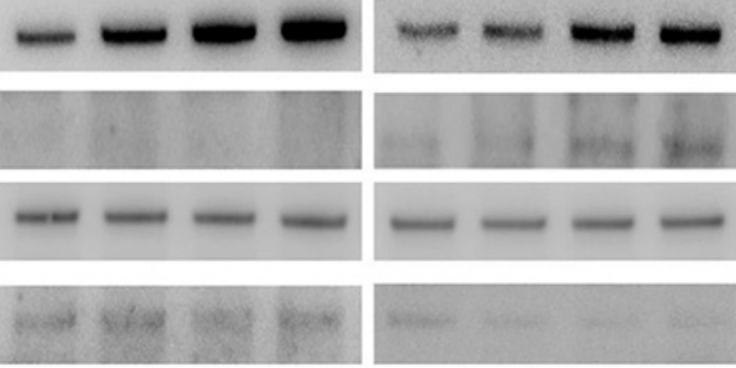

SIRT1

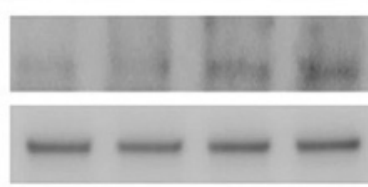

Ac-p65

p65

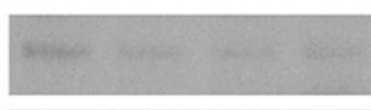

Ac-p53
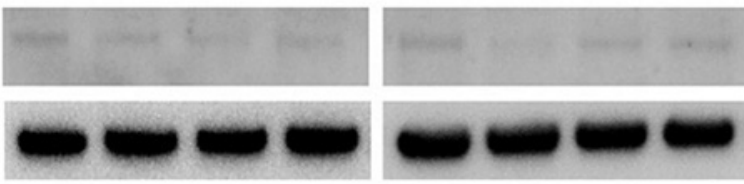

p53

GAPDH

Fig. 3. $\mathrm{NF}-\kappa \mathrm{B} / \mathrm{p} 53 / \mathrm{p} 21$ pathway is involved in IL-1 $\beta$-induced senescence of VSMCs. A Immunoblotting analysis for IL-1 $\beta$-activated NF- $\mathrm{kB} / \mathrm{p} 53 / \mathrm{p} 21$ pathway. VSMCs were treated with phosphate or phosphate/IL-1 $\beta$ or phosphate/IL-1 $\beta /$ anti-IL-1 $\beta$ neutralization antibody over 3 days. B Immunoblotting analysis for inhibition of the $\mathrm{NF}-\kappa \mathrm{B} /(\mathrm{SIRT}) / \mathrm{p} 53 / \mathrm{p} 21$ pathway in VSMCs treated with resveratrol. VSMCs were cultured in medium containing resveratrol with either phosphate alone or phosphate/IL-1 $\beta$ over 3 days. Pi, phosphate; $\mathrm{Ab}$, anti-IL-1 $\beta$ neutralization antibody; Res, resveratrol; 3-day vehicle denotes 3-passage VSMCs cultured in complete DMEM/F-12 medium for 3 days; SIRT1, sirtuin-1; IL, interleukin. 
A

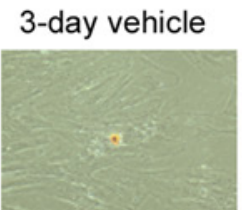

$1 d$

$2 \mathrm{~d}$

$3 d$
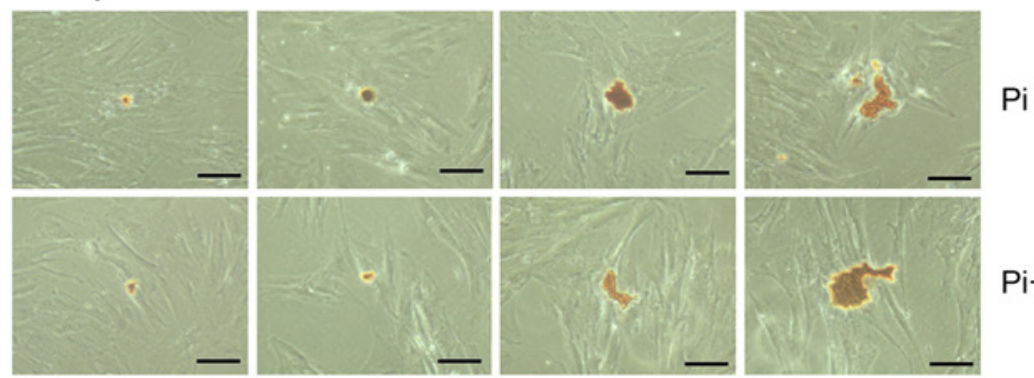

$\mathrm{Pi}+\mathrm{IL}-1 \beta$
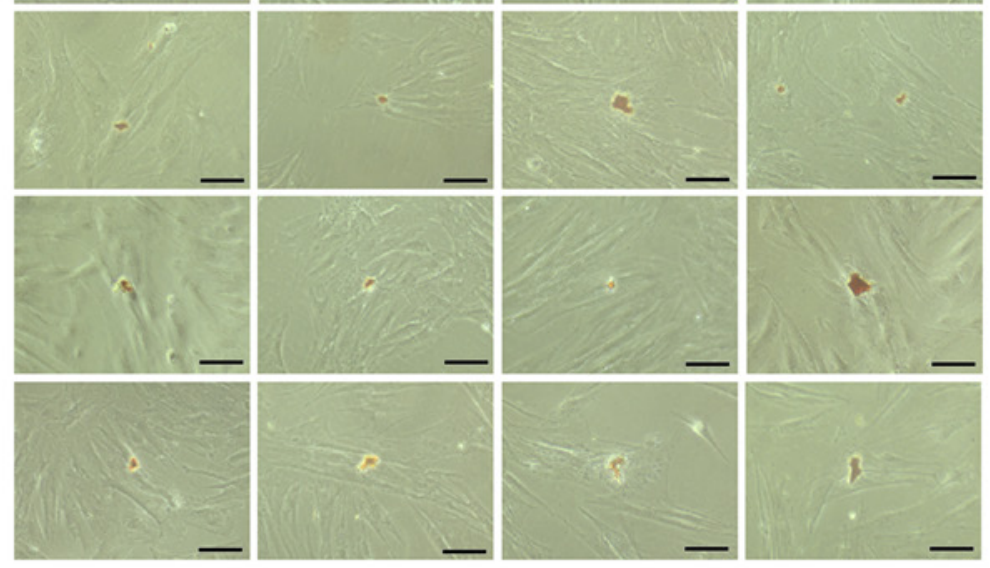

$P i+I L-1 \beta+A b$
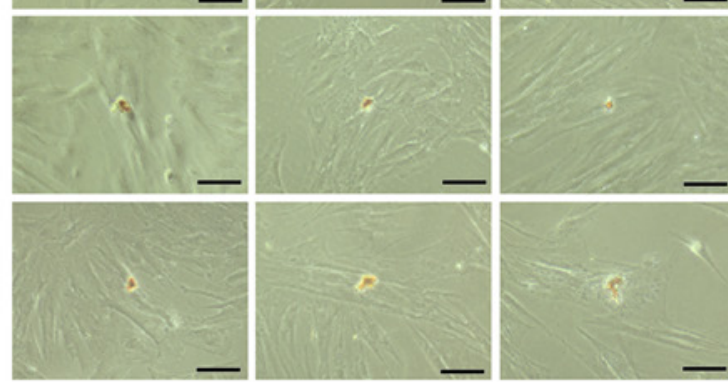

$\mathrm{Pi}+\mathrm{Res}$

Pi+IL-1 $1 \beta+R e s$

B

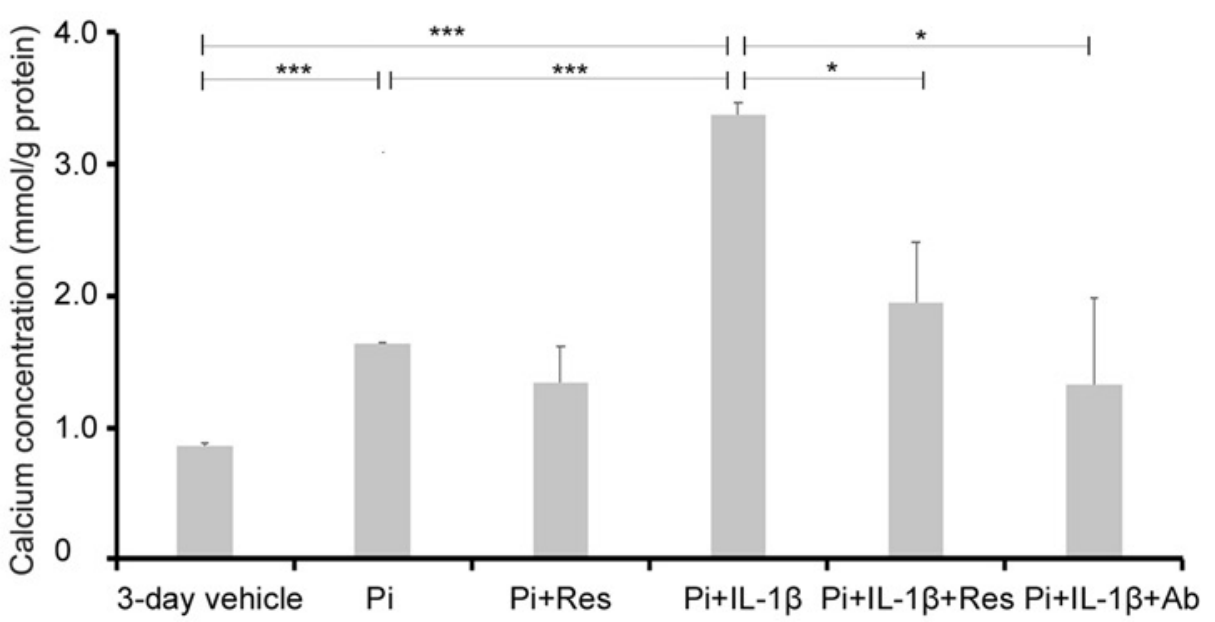

Fig. 4. IL-1 $\beta$ induces senescence-associated calcification in VSMCs. A Alizarin red staining for the detection of mineral deposition in VSMCs (Scale bar $=100 \mu \mathrm{m}$ ). Alizarin red staining for the detection of phosphateinduced or IL-1 $\beta$-induced mineral deposition in VSMCs by exposure to resveratrol, phosphate/IL-1 $\beta /$ resveratrol or phosphate/IL-1 $\beta$ /anti-IL-1 $\beta$ neutralization antibody (Scale bar $=100 \mu \mathrm{m}$ ). $\mathbf{B}$ The colorimetric analysis of calcium content versus total protein (mmol/g protein) in 3-passage VSMCs treated with phosphate, phosphate/IL-1 $\beta$, phosphate/resveratrol, phosphate/IL-1 $\beta /$ resveratrol, or phosphate/IL-1 $\beta /$ anti-IL-1 $\beta$ neutralization antibody for 3 days. Data were presented as the mean \pm SD of triplicate experiments $\left(^{* * *} p<\right.$ $0.001, * p<0.05)$. Pi, phosphate; Ab, anti-IL-1 $\beta$ neutralization antibody; Res, resveratrol; 3-day vehicle means 3-passage VSMCs cultured in complete DMEM/F-12 medium for 3 days. IL, interleukin. 
Fig. 5. Resveratrol represses osteoblastic transition in IL-1 $\beta$ induced senescent VSMCs. A Immunoblotting analysis for expression of p21, a-SMA, RUNX2, and BMP2 in VSMCs by exposure to phosphate or phosphate/resveratrol over 3 days. B Immunoblotting analysis for the expression of p21, a-SMA, RUNX2, and BMP2 in VSMCs cultured in medium with phosphate/IL-1 $\beta$ or phosphate/ IL-1 $\beta /$ resveratrol over 3 days. $\mathrm{Pi}$, phosphate; $A b$, anti-IL-1 $\beta$ neutralization antibody; Res, resveratrol; 3-day vehicle denotes 3-passage VSMCs cultured in complete DMEM/F-12 medium for 3 days; a-SMA, $\alpha$-smooth muscle actin; RUNX2, runt-related transcription factor-2; BMP2, bone morphogenetic protein-2; IL, interleukin.

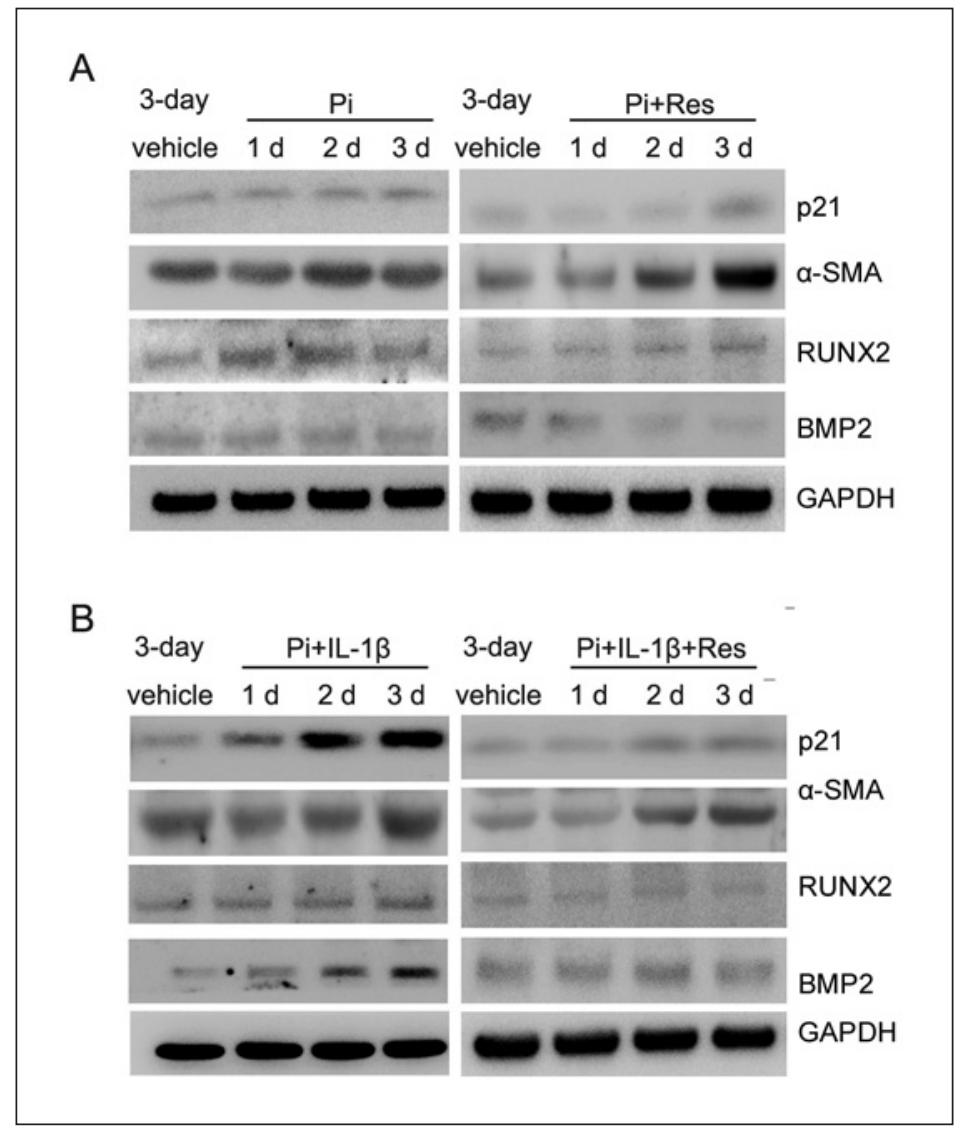

\section{Discussion}

There are some pieces of evidence that support that vascular calcification occurs due to senescence-associated osteoblastic transition in VSMCs, including in vivo experiments in animal models with chronic kidney injury and in vitro experiments [16, 23, 24]; however, not much is known about the direct evidence to support the correlations between senescent VSMCs and vascular calcification caused by osteoblastic transdifferentiation in human arteries from the patients with ESRD. As a senescent marker, p21 is widely expressed in the senescent cells. The expression of BMP2 and RUNX2 is also observed, while osteoblastic transition occurs in VSMCs. In the present study, immunohistochemistry analysis and Alizarin red staining results showed that $73.3 \%$ of patients with ESRD exhibited the expression of BMP2 and p21 in calcified vascular muscles. This suggests that there may be a molecular coordination between vascular calcification and cellular senescence associated with osteoblastic transition in VSMCs.

The characteristics of premature senescent phenotypes include cellular morphology alternation, telomere erosion, cell growth arrest, and increased SA- $\beta$-gal activity. To remove spontaneous senescence, young VSMCs (3-passage) were treated only 3 days in the present study. Considering that loading phosphate is mainly responsible for generating senescencelike phenotypes in VSMCs in in vivo or in vitro studies [20,25-28], young VSMCs were treated for only 3 days with loading phosphate alone to confirm whether it induces senescence. However, based on cellular morphology, p21 expression, and SA- $\beta$-Gal activity, overloading of phosphate failed to promote the senescence of VSMCs within 3 days. The possible reason 


\section{Kidney \\ Blood Pressure \\ Research}

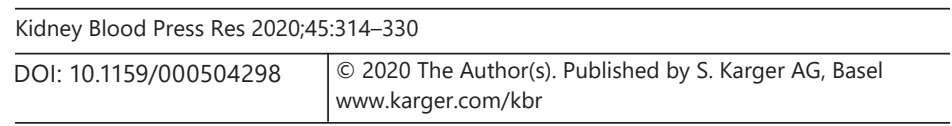

Han et al.: IL-1 $\beta$-Induced Senescence Promotes Osteoblastic Transition of VSMCs

is that young VSMCs (3-passage cells) were in vitro treated with phosphate in a short time (3 days).

It has been reported that IL-1 $\beta$ may induce vascular calcification in patients with CKD or ESRD $[28,29]$. Increased levels of cytokines are associated with increased cardiovascular risks and mortality in these patients due to chronic inflammation and its consequences [30, 31]. The study by Dai et al. [14] proved that human and rat osteoarthritic chondrocytes exposed to IL-1 $\beta$ display the features of premature senescent phenotypes. As expected, the features of premature senescent phenotypes, including cellular morphology alternation, increased SA- $\beta$-Gal activity, increased levels of ROS and increased p21 expression, were observed in human primary VSMCs exposed to IL- $1 \beta$ over 3 days. These results strongly supported IL-1 $\beta$-induced senescent VSMCs. Coincident with increased p21 expression, activated NF- $\mathrm{kB}$ (p-p65), Ac-p65, p53, and Ac-p53 were markedly increased in VSMCs by exposure to IL-1 $\beta$. Additionally, SIRT1, which exerts a protective role in cellular senescence [32], was not significantly altered in VSMCs exposed to IL-1 $\beta$ over a 3-day period, as it was reported that activated NF- $\kappa B$ inhibits SIRT1 expression [22]. It is well known that resveratrol is one of the most functional activators of SIRT1, and it is widely used as an antiaging agent. After treatment with resveratrol, VSMCs exhibited a decreased SA- $\beta$-Gal activity and decreased p21 expression, coincident with increased SIRT1 expression. It is indicated that resveratrol retards IL-1 $\beta$-induced senescence in VSMCs depending upon upregulated SIRT1 expression. Furthermore, as a member of the sirtuin family of nicotinamide adenine dinucleotide-dependent deacetylases, SIRT1 can deacetylate many different transcription factors in the nucleus such as p53 and NF-KB $[32,33]$. Immunoblotting showed that decreased Ac-p65 and Ac-p53 were associated with the increased SIRT1 expression in VSMCs by exposure to resveratrol. Mechanistically, the IL-1 $\beta$-induced senescent phenotype was partially associated with the activation of NF- $\mathrm{KB} / \mathrm{p} 53 / \mathrm{p} 21$ pathways.

For several years, it is well known that a role of the inflammasome as an IL-1 $\beta$-activating platform in the process of vascular calcification [28,34]. Though the high concentration of plasma phosphate may be not sufficient to induce vascular calcification [35], a recent study proved that an anti-IL-1 $\beta$ antibody represses vascular calcification in a mouse model of familial hypercholesterolemia [8]. Phosphate-induced VSMC calcification, by increasing oxidative stress and osteoblastic differentiation, results in the promotion of altered osteoblastic phenotype in VSMCs [36]. Although we found cardiovascular calcification were not associated with serum levels of IL-1 $\beta$ in ESRD patients, it is reported that the deposition of basic calcium phosphate crystals in macrophage induces the release of IL-1 $\beta$ [37]. In concord with positive staining of BMP2, p21, and mineral deposition, IL-1 $\beta$ highly expressed in the distal radial arteries from 8 patients with ESRD in the current study. Furthermore, as reported previously, high glucose levels promote mouse and human VSMC transdifferentiation into chondrocytes possibly through IL-1 $\beta$ activation [38]. These findings may shed light on the role of the inflammasome as an IL-1 $\beta$-inducing effector in the process of vascular calcification. Based on the Alizarin red staining and calcium content detection, we found that IL-1 $\beta$ promoted vascular calcification in VSMCs. Similar to a previous report [36], high phosphate enhanced the expression of RUNX2 (but not BMP2), which is highly expressed in osteoblasts. Interestingly, the expression of BMP2, rather than RUNX2, was dramatically increased in VSMCs exposed to IL-1 $\beta$. This suggests that IL- $1 \beta$ contributes to osteoblastic transdifferentiation in a BMP2-dependent manner, whereas high phosphate induces an osteoblastic transition in a RUNX2-dependent manner.

We demonstrated that IL-1 $\beta$-inducing senescent phenotype might be required for the activation of NF- $\kappa B / p 53 / p 21$ pathways. IL-1 $\beta$-activating NF- $\kappa B$ suppressed the expression of SIRT1, an endogenous inhibitor of senescence in VSMCs. Alizarin red staining showed both hyperphosphate-induced and IL- $1 \beta$-induced vascular calcification was markedly inhibited in VSMCs exposed to either resveratrol or the anti-IL-1 $\beta$ neutralization antibody. Resveratrol 
Fig. 6. IL-1 $\beta$-activated and resveratrol-inhibited the NF- $\kappa \mathrm{B} / \mathrm{p} 53 / \mathrm{p} 21$ pathways in VSMCs. Res, resveratrol; SIRT1, sirtuin-1; VSMC, vascular smooth muscle cell; IL, interleukin.

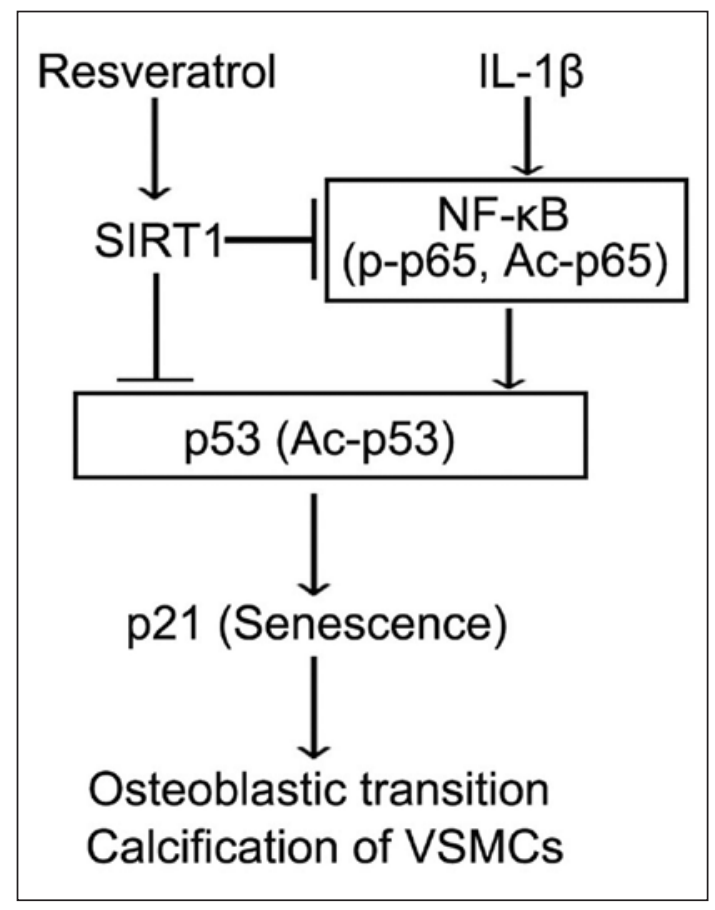

dramatically decreased the expression of RUNX2 and BMP2 in VSMCs cultured in calcification medium, whereas it did not affect the expression of RUNX2 and BMP2 in VSMCs exposed to IL-1 $\beta$ and hyperphosphate. Resveratrol treatment may have secondary effects on osteoblastic transition in VSMCs, as it caused a reduction of p-p65 and Ac-p65 through an increased SIRT1 expression $[33,39,40]$. Therefore, SIRT1 exerts a protective role in cellular senescence through the inhibition of p21 expression in VSMCs and is associated with crosstalk between the osteoblastic transition and cellular senescence (Fig. 6).

Taken together, IL-1 $\beta$, which highly expressed in medial smooth muscles of arteries from patients with ESRD, may be an inducer of vascular calcification in patients with ESRD. Higher levels of IL-1 $\beta$ induce senescence in VSMCs through the activation of the NF- $\kappa B / p 53 / p 21$ pathway and contribute to vascular calcification caused by osteoblastic transition in the senescent VSMCs. Although effective therapies to treat and prevent vascular calcification in clinical settings are not available; yet, our findings suggest that resveratrol would be helpful to prevent the senescence-associated medial calcification owing to the osteoblastic transition of VSMCs during senescence.

\section{Acknowledgments}

We thank Dr. Xijun Gong and Huan Li from the Department of Clinical Image in the Second Affiliated Hospital of Anhui Medical University for helping read the clinical images. This work was supported by the National Natural Science Foundation of China (grant number 81772909).

\section{Statement of Ethics}

The authors have no ethical conflicts to disclose. 


\section{Kidney \\ Blood Pressure \\ Research}

\begin{tabular}{l|l}
\hline Kidney Blood Press Res 2020;45:314-330 \\
\hline DOI: 10.1159/000504298 & $\begin{array}{l}\text { @ 2020 The Author(s). Published by S. Karger AG, Basel } \\
\text { www.karger.com/kbr }\end{array}$ \\
\hline
\end{tabular}

Han et al.: IL-1ß-Induced Senescence Promotes Osteoblastic Transition of VSMCs

\section{Disclosure Statement}

The authors have no conflicts of interest to declare.

\section{Author Contributions}

H.Z. and D.W. conceived and designed the experiments. L.H., Y.Z., M.Z., L.G., and J.W. performed the experiments. F.Z. and D.X. analyzed the data. Y.Z., L.G., and D.W. interpreted the data contributed to the manuscript structure and flow. Y.Z., M.Z., Z.Y., Y.X., and D.W. collected experimental specimens. L.H. and H.Z. wrote the paper. All authors reviewed and confirmed the manuscript.

\section{References}

1 Demer LL, Tintut Y. Vascular calcification: pathobiology of a multifaceted disease. Circulation. 2008 Jun; 117(22):2938-48.

2 Mohr W, Görz E. [Granular media calcinosis of the aorta. Structural findings, historical review and pathogenetic significance]. Z Kardiol. 2001 Dec;90(12):916-28.

3 Shroff RC, McNair R, Figg N, Skepper JN, Schurgers L, Gupta A, et al. Dialysis accelerates medial vascular calcification in part by triggering smooth muscle cell apoptosis. Circulation. 2008 0ct;118(17):1748-57.

4 Tomiyama C, Carvalho AB, Higa A, Jorgetti V, Draibe SA, Canziani ME. Coronary calcification is associated with lower bone formation rate in CKD patients not yet in dialysis treatment. J Bone Miner Res. 2010 Mar;25(3): 499-504.

5 Agharazii M, St-Louis R, Gautier-Bastien A, Ung RV, Mokas S, Larivière R, et al. Inflammatory cytokines and reactive oxygen species as mediators of chronic kidney disease-related vascular calcification. Am J Hypertens. 2015 Jun;28(6):746-55.

6 Kleemann R, Zadelaar S, Kooistra T. Cytokines and atherosclerosis: a comprehensive review of studies in mice. Cardiovasc Res. 2008 Aug;79(3):360-76.

7 Shobeiri N, Bendeck MP. Interleukin-1 $\beta$ Is a Key Biomarker and Mediator of Inflammatory Vascular Calcification. Arterioscler Thromb Vasc Biol. 2017 Feb;37(2):179-80.

8 Awan Z, Denis M, Roubtsova A, Essalmani R, Marcinkiewicz J, Awan A, et al. Reducing Vascular Calcification by Anti-IL-1 $\beta$ Monoclonal Antibody in a Mouse Model of Familial Hypercholesterolemia. Angiology. 2016 Feb; 67(2):157-67.

9 Ghosh S, Karin M. Missing pieces in the NF-kappaB puzzle. Cell. 2002 Apr;109(2 Suppl):S81-96.

10 Hénaut L, Sanz AB, Martin-Sanchez D, Carrasco S, Villa-Bellosta R, Aldamiz-Echevarria G, et al. TWEAK favors phosphate-induced calcification of vascular smooth muscle cells through canonical and non-canonical activation of NFKB. Cell Death Dis. 2016 Jul;7(7):e2305.

11 Zhao CQ, Wang LM, Jiang LS, Dai LY. The cell biology of intervertebral disc aging and degeneration. Ageing Res Rev. 2007 Oct;6(3):247-61.

12 Yang W, Yu XH, Wang C, He WS, Zhang SJ, Yan YG, et al. Interleukin-1beta in intervertebral disk degeneration. Clin Chim Acta. 2015 Oct; 450:262-72.

13 So MW, Lee EJ, Lee HS, Koo BS, Kim YG, Lee CK, et al. Protective effects of ginsenoside Rg3 on human osteoarthritic chondrocytes. Mod Rheumatol. 2013 Jan;23(1):104-11.

14 Dai SM, Shan ZZ, Nakamura H, Masuko-Hongo K, Kato T, Nishioka K, et al. Catabolic stress induces features of chondrocyte senescence through overexpression of caveolin 1: possible involvement of caveolin 1-induced down-regulation of articular chondrocytes in the pathogenesis of osteoarthritis. Arthritis Rheum. 2006 Mar; 54(3):818-31.

15 Shanahan CM, Cary NR, Salisbury JR, Proudfoot D, Weissberg PL, Edmonds ME. Medial localization of mineralization-regulating proteins in association with Mönckeberg's sclerosis: evidence for smooth muscle cellmediated vascular calcification. Circulation. 1999 Nov;100(21):2168-76.

16 Zhou Y, Wang JY, Feng H, Wang C, Li L, Wu D, et al. Overexpression of c1q/tumor necrosis factor-related protein-3 promotes phosphate-induced vascular smooth muscle cell calcification both in vivo and in vitro. Arterioscler Thromb Vasc Biol. 2014 May;34(5):1002-10.

17 Lin ME, Chen T, Leaf EM, Speer MY, Giachelli CM. Runx2 Expression in Smooth Muscle Cells Is Required for Arterial Medial Calcification in Mice. Am J Pathol. 2015 Jul;185(7):1958-69.

18 Sowa AK, Kaiser FJ, Eckhold J, Kessler T, Aherrahrou R, Wrobel S, et al. Functional interaction of osteogenic transcription factors Runx2 and Vdr in transcriptional regulation of Opn during soft tissue calcification. Am J Pathol. 2013 Jul;183(1):60-8. 


\section{Kidney \\ Blood Pressure \\ Research}

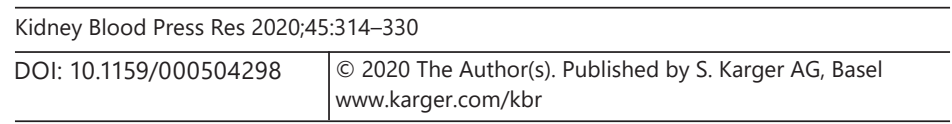

Han et al.: IL-1 $\beta$-Induced Senescence Promotes Osteoblastic Transition of VSMCs

19 Tu Z, Zhang S, Zhou G, Zhou L, Xiang Q, Chen Q, et al. LMO4 Is a Disease-Provocative Transcription Coregulator Activated by IL-23 in Psoriatic Keratinocytes. J Invest Dermatol. 2018 May;138(5):1078-87.

20 Xu D, Zeng F, Han L, Wang J, Yin Z, Lv L, et al. The synergistic action of phosphate and interleukin-6 enhances senescence-associated calcification in vascular smooth muscle cells depending on p53. Mech Ageing Dev. 2019 Sep;182:111124.

21 Zeng Y, Wang PH, Zhang M, Du JR. Aging-related renal injury and inflammation are associated with downregulation of Klotho and induction of RIG-I/NF- $\kappa B$ signaling pathway in senescence-accelerated mice. Aging Clin Exp Res. 2016 Feb;28(1):69-76.

22 Kauppinen A, Suuronen T, Ojala J, Kaarniranta K, Salminen A. Antagonistic crosstalk between NF- $\kappa B$ and SIRT1 in the regulation of inflammation and metabolic disorders. Cell Signal. 2013 Oct;25(10):1939-48.

23 Dayanand P, Sandhyavenu H, Dayanand S, Martinez J, Rangaswami J. Role of Bisphosphonates in Vascular calcification and Bone Metabolism: A Clinical Summary. Curr Cardiol Rev. 2018;14(3):192-9.

24 Martín-Pardillos A, Sorribas V. Effects of donor age and proliferative aging on the phenotype stability of rat aortic smooth muscle cells. Physiol Rep. 2015 Nov;3(11):e12626.

25 Quarles LD. Endocrine functions of bone in mineral metabolism regulation. J Clin Invest. 2008 Dec;118(12): 3820-8.

26 Kuro-o M, Matsumura Y, Aizawa H, Kawaguchi H, Suga T, Utsugi T, et al. Mutation of the mouse klotho gene leads to a syndrome resembling ageing. Nature. 1997 Nov;390(6655):45-51.

27 Kurosu H, Yamamoto M, Clark JD, Pastor JV, Nandi A, Gurnani P, et al. Suppression of aging in mice by the hormone Klotho. Science. 2005 Sep;309(5742):1829-33.

28 Razzaque MS, Sitara D, Taguchi T, St-Arnaud R, Lanske B. Premature aging-like phenotype in fibroblast growth factor 23 null mice is a vitamin D-mediated process. FASEB J. 2006 Apr;20(6):720-2.

29 Amdur RL, Feldman HI, Gupta J, Yang W, Kanetsky P, Shlipak M, et al.; CRIC Study Investigators. Inflammation and Progression of CKD: the CRIC Study. Clin J Am Soc Nephrol. 2016 Sep;11(9):1546-56.

30 Kimmel PL, Phillips TM, Simmens SJ, Peterson RA, Weihs KL, Alleyne S, et al. Immunologic function and survival in hemodialysis patients. Kidney Int. 1998 Jul;54(1):236-44.

31 Weiner DE, Tighiouart H, Elsayed EF, Griffith JL, Salem DN, Levey AS, et al. Inflammation and cardiovascular events in individuals with and without chronic kidney disease. Kidney Int. 2008 Jun;73(12):1406-12.

32 Vaziri H, Dessain SK, Ng Eaton E, Imai SI, Frye RA, Pandita TK, et al. hSIR2(SIRT1) functions as an NADdependent p53 deacetylase. Cell. 2001 Oct;107(2):149-59.

33 Yeung F, Hoberg JE, Ramsey CS, Keller MD, Jones DR, Frye RA, et al. Modulation of NF-kappaB-dependent transcription and cell survival by the SIRT1 deacetylase. EMBO J. 2004 Jun;23(12):2369-80.

34 Johnson K, Hashimoto S, Lotz M, Pritzker K, Terkeltaub R. Interleukin-1 induces pro-mineralizing activity of cartilage tissue transglutaminase and factor XIIIa. Am J Pathol. 2001 Jul;159(1):149-63.

35 Murshed M, Harmey D, Millán JL, McKee MD, Karsenty G. Unique coexpression in osteoblasts of broadly expressed genes accounts for the spatial restriction of ECM mineralization to bone. Genes Dev. 2005 May; 19(9):1093-104.

36 Lee KM, Lee EO, Lee YR, Joo HK, Park MS, Kim CS, et al. APE1/Ref-1 Inhibits Phosphate-Induced Calcification and Osteoblastic Phenotype Changes in Vascular Smooth Muscle Cells. Int J Mol Sci. 2017 Sep;18(10):E2053.

37 Nadra I, Mason JC, Philippidis P, Florey O, Smythe CD, McCarthy GM, et al. Proinflammatory activation of macrophages by basic calcium phosphate crystals via protein kinase $\mathrm{C}$ and MAP kinase pathways: a vicious cycle of inflammation and arterial calcification? Circ Res. 2005 Jun;96(12):1248-56.

38 Bessueille L, Fakhry M, Hamade E, Badran B, Magne D. Glucose stimulates chondrocyte differentiation of vascular smooth muscle cells and calcification: A possible role for IL-1ß. FEBS Lett. 2015 Sep;589(19PartB 19 Pt B):2797-804.

39 Holmes-McNary M, Baldwin AS Jr. Chemopreventive properties of trans-resveratrol are associated with inhibition of activation of the IkappaB kinase. Cancer Res. 2000 Jul;60(13):3477-83.

40 Zhang Y, Chen F. Reactive oxygen species (ROS), troublemakers between nuclear factor-kappaB (NF-kappaB) and c-Jun NH(2)-terminal kinase (JNK). Cancer Res. 2004 Mar;64(6):1902-5. 